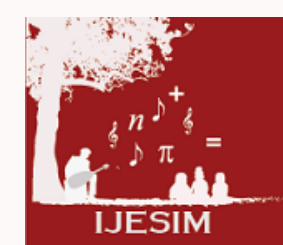

International Journal of Educational Studies in Mathematics

ISSN: 2148 - 5984

wWw.ijesim.com

\title{
A Mathematician's Influence on Students' Perceptions - The Case of Mathematical Induction
}

\author{
Amal Sharif-Rasslan'; Shaker Rasslan² \\ ${ }^{1}$ The Academic Arab College for Education, Haifa, Israel, \\ ${ }^{2}$ Al-Qasemi Academic College of Education, Israel
}

ABSTRACT

\begin{abstract}
In this study, we report on a professor of mathematics who also teaches mathematics in a secondary school. We checked the influence of the professor's presenting the mathematical induction (MI) principle on his students' perceptions of the principle. Extensive observations on the MI content were performed to determine the professor's conception the MI principle. Moreover, seventy four students answered a questionnaire which was built to examine the students' perceptions of the MI principle. Finally, both data analysis were triangulated and crosschecked. It was concluded that the students' main perception of MI was: mathematical induction identifies the natural number set, including formulation; following four representations of the MI that were exposed by the teacher. This means that the learners mainly, perceived MI by reflecting on the properties of the set of natural numbers, in addition to the usual perception which is as "a method of proof". Consequently, it can be argued that this representation has pedagogical advantages.
\end{abstract}

Keywords:

mathematical induction principle, perceptions, secondary school, peano's axiom, infinity.

(c) 2015 IJESIM. All rights reserved

Received 08.12.2014 Received in revised form 05.07.2015 Accepted 25.09.2015 Available online 20.10.2015

\section{Introduction and Theoretical Background}

Mathematics is a subject which has to be dealt with a rigorous way, but the manner in which we represent it may affect the learner. It is important to discover some methods of teaching/doing mathematics emphasizing the accuracy and rigorous of mathematics. The first author checked matriculation examinations in mathematics for ten years. Every year, some examinations were found exceptional. These examinations' results were described rigorously and in a fully reasoned way. It was discovered that the teacher of those particular students was a university professor who was carrying extensive research in pure mathematics, and at the same time had also taught in a secondary school for about twenty years. Consequently, this case was investigated thoroughly in different aspects: the teacher's way of teaching, the impact of doing mathematical research on teaching elementary mathematics at school and the influence of the teacher on his students' perception a mathematical concept.

In this paper we shall concentrate on the MI principle, investigating this content from the view of the mentioned professor and from the view of his students, trying to emphasize the influence of the teacher on his students' perception the MI principle.

\footnotetext{
${ }^{1}$ Corresponding author's address: The Academic Arab College for Education, 22 Hahashmal St., P.O.Box 8349, Haifa 33145, Israel

Telephone: + $97248303500 /+972522411329$

Fax: +97248233517

e-mail: amalras@macam.ac.il

DOI: http://dx.doi.org/10.17278/ijesim.2015.01.001
} 
Mathematical induction principle is a basic element in the secondary school curriculum in mathematics. It has been argued that MI principle should be introduced in upper secondary school (NCTM, 2000) as the students' understanding of MI principle may facilitate their understanding inductive reasoning. There are two ways of presenting the MI principle content: (a) as a statement: "For each $\mathrm{n} \in \mathrm{N}$, let $\mathrm{Pn}$ be a mathematical statement or proposition. Suppose that (i) P0 (or P1) is true; and (ii) if Pk is true for any k $\in \mathrm{N}$, then $\mathrm{Pk}+1$ is true too. Then Pn is true for all $\mathrm{n} \in \mathrm{N}$." (Hall, 1971, p. 48), and (b) in set language: "If a subset $\mathrm{S}$ of $\mathrm{N}$ contains 1 and contains the successor of each of its members, then it contains (and equals) all of $\mathrm{N}^{\prime \prime}$ (Maxfield et al., 1971, p. 21).

In this paper, we refer to MI principle in its second presentation, emphasizing that the significance of MI principle goes beyond its role as a proof method; moreover, emphasizing its power to reveal the structure of the set of natural numbers.

Henkin (1961) suggested that the set-theoretical formulation has pedagogical advantages. Many studies have been carried out on this concept; most of these dealt with the difficulties of the learners in attempting to relate to the MI principle; moreover many of the studies referred to the true statement version (Dubinsky, 1986; Dubinsky, 1990; Ernest, 1984; Fischbein and Angel, 1989; Lowenthal and Eisenberg, 1992; MovshovitzHadar, 1993; Harel and Sowder, 1998; 2007; Harel, 2002; Palla et al., 2012). Movshovitz-Hadar (1993) urged that MI (in its sentential-logic expression) is a complex topic. Ernest (1984) devised a conceptual analysis of mathematical induction, which "provides a theoretical foundation on which the teaching of the method can be erected" (p.180). Ernest (1984) added: "On the one hand, the inductive method is a heuristic method for arriving at the conjectured generality describing a finite sequence of example. On the other hand, mathematical induction is a rigorous form of deductive proof" (p. 181).

Harel and Sowder $(1998,2007)$ describes three classes of proof schemes: the external conviction proof scheme- this scheme depends on the authority of the teacher or the textbook or on symbols, the empirical proof scheme- this scheme bases on evidence from example or/and measurements, and the deductive proof scheme which relies on logical inference rules. Within this framework, Harel (2002) considered recursiveness as an important dimension of MI principle. He claimed that, when students face recursion problems, they are induced to focus on process pattern generalization rather than result pattern generalization; which is a way of thinking that characterizes the structure of MI principle. Later, Pedemonte (2007) recognized the process pattern generalization way of thinking as a requirement to construct a proof based on MI principle. Palla et al. (2012) investigated the meaning students attribute to the structure of MI and the process of proof construction using $\mathrm{MI}$ in the context of a geometric recursion problem; they identified two types of transition from argumentation to proof: In the first type MI principle was applied to the algebraic statement that derived from the direct translation of the geometric situation. In the second type, MI principle was embedded functionally in the geometric structure of the pattern.

A teacher's role in mathematics lessons is seen primarily as an activator of pupils' thinking skills, to help pupils to understand mathematical structures and ideas as well as to help pupils when developing their own mathematical knowledge (Schoenfeld, 1985; Sfard, 1998). Sfard (2001), claims that "a student may have an immediate object-level goal of solving a mathematical problem that is embedded into the long-term goal of learning some new mathematics". The teacher's practical knowledge of a concept can be discovered by the cognitive schema of the concept in his mind (the cognitive schema of a concept refers to the cluster of the different meanings/representations of the concept; in Hiebert \& Carpenter (1992) it is called a "network"). Information can be stored in long term memory (LTM) only after processed (Silver, 1987); so LTM is a special place to check the knowledge of the student, without forgetting that short term memory (STM) is the place where most of the cognitive actions take place (Silver, 1987). Consequently, to investigate the influence of the teacher's conceptions on the students' perceptions about a given concept, it is worthy to concentrate on the teaching-learning process and check the knowledge of the students by examining the students' STM and LTM of the concept.

The aim of this study is to check the influence of the teacher's conception of MI principle on his students' perception the concept. The following two main questions were derived from this aim:

1. How do students, who have learned the "mathematical induction" principle in the set language, perceive the principle? 
2. How do the students' perceptions relate to the teacher's perception of the principle?

\section{Methods}

This study is a qualitative case study. It is mainly focusing on answering "how" questions (Yin, 2003).

\section{Participants}

This case study includes two kinds of participants:

I. A university professor: he was carrying extensive research in pure mathematics, taught/teaches mathematical courses for graduate and undergraduate students more than thirty years, and at the same time had also taught in a secondary school for about twenty years. As a university professor, this professor was known as a regular lecturer in the sense that he performs standard teaching and related mathematics faculty duties. As a researcher, he was known as an active and had several fields of expertise especially in geometric functions, mathematical statistics and analytical probability.

II. Seventy four students: 33 students were in 12th grade, and learned the MI principle approximately one year before the research was carried out; and 41 students were in 11th grade, and learned MI principle during the period of this research. All the students who participated in this study, learned in the same school, which is placed in small Arab city in the north of Israel. All these students, learned mathematics as a high level, at least seven hours per week. Most of these students want to pursue an undergraduate degree in scientific subjects.

\section{Data}

To answer the questions we employed two sets of data:

-The first set focused on the teacher, following the teaching process.

-The second set focused on the students, trying to get data related to the learning process.

\section{Teacher's data}

Extensive data were collected on the teacher; he was observed, videotaped for approximately 385 minutes on the subject of "mathematical induction" (It was the fulltime the teacher taught the subject). The aim of this form of data, was to follow the teaching process and discover the teacher's conception of the MI principle.

\section{Students' data}

A 3-question questionnaire, relating to the subject of "mathematical induction", was compiled, and administered to two classes of the professor teacher. One class (containing 33 students, 12th grade) learned the subject approximately one year before its students answered the questionnaire; the students in the other class $(41,11$ th grade) answered it during the period in which they studied the subject. The aim of this kind of data, was to check the students' STM and LTM of the MI principle.

The questionnaire: Assuming that students should be familiar with mathematical contents in the form they learn in their class, the questionnaire included questions that reflect the way they were taught by the professor teacher. It should be noted that these questions were written after the observations in the professor's discourse, during the period he was teaching the concept of "mathematical induction"; thus the questions were composed in a way that fits the spirit of the observed lessons.

For validity of the questionnaire, it was given to 10 students who did not participate in current study and was examined by the professor teacher.

The questionnaire included the following questions:

Question 1: Mathematical induction relates to natural numbers as:
a. The sum operation
b. The multiplication operation
c. Order
d. The division operation

Question 2: Mathematical induction
a. defines the set of natural numbers
c. orders the set of natural numbers
b. characterizes the set of natural numbers
d. all the answers are wrong 
Question 3: What is "mathematical induction"?

The aim of the first two questions in the questionnaire was to check the students' conceptualization of mathematical induction, and the aim of the third question was to confirm the results obtained from the first two questions.

\section{Analysis and Results}

\section{The teaching process: Teacher's data analysis}

All the professor's MI lessons were observed, and the data were divided according to the professor's perceptions of MI. Clustering the different meanings, representations and basic concepts which are related to MI principle, according the lessons of the professor teacher, a semantic net was devised to illustrate the professor's perception of the MI principle. This semantic net is a cognitive schema which explains and clarifies the teacher's conception the MI principle, Figure 1.

The result is shown in Figure 1.

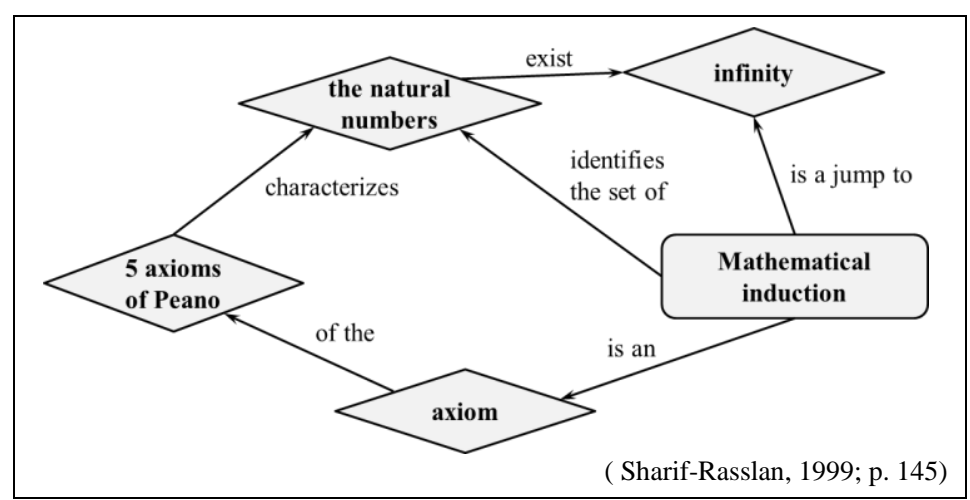

Figure 1: Mathematical induction from the perspective of a professional mathematician.

The main perceptions of the "mathematical induction" principle in the professor's mind as described in figure 1 are:

1. "Mathematical induction" is a jump to infinity;

2. "Mathematical induction" is one axiom of the five axioms of Peano;

3. "Mathematical induction" identifies the set of natural numbers.

The formulation of the mathematical induction principle is a fourth perception; our subject (the professor) used the formulation of MI principle in the set theory language.

For each perception of the MI principle, we determined a weight. The weight of each perception in each lesson was calculated as the ratio of all the statements (that were used/said by the professor during the lesson) to the total number of statements in the same lesson, figure 2. For example, in the first lesson the teacher emphasized the perception that depends on infinity more than identification of the natural numbers set, but he referred to the Peano's axioms occasionally and did not refer to the formulation of the principle; this is described in figure 2(a). While during the fourth lesson the professor emphasized only the formulation of the MI principle, figure 2(d).

By analogy to the law of gravity in physics, it is clear that the heaviest weight is below and lightest is above, figure 2(a); we have to notice that the sum of all the ratios in figure 2(a) is less than 1, this difference is due to the statements which are not related to the content area. Similarly, figures 2(b), 2(c) and 2(d) describe the representation in the rest three lessons. Notice that the teacher emphasized the "infinity" perception of MI in the first two lessons, and it was negligible in the rest lessons; the "identification" perception was emphasized in the third lesson, the "formulation" perception approached 1 in the fourth lesson, and it was negligible in the first two lessons. 


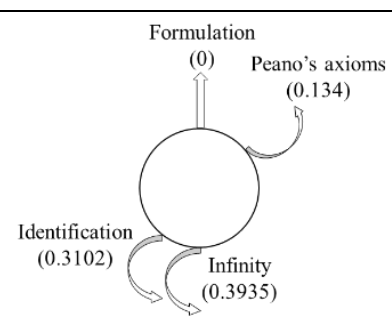

(a)

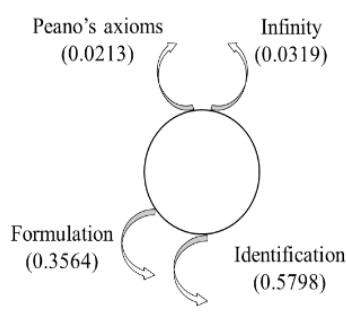

(c)

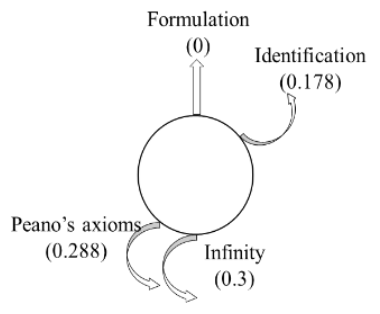

(b)

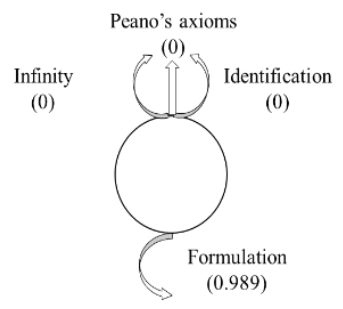

(d)

Figure 2: Description of the teacher's representation of the MI principle as a function of the lessons.

Table 1 summarizes the total references of the teacher to each perception of the MI principle during the four lessons; in other words, table 1 provide the ratio between all the statements that refer to some perception and between all the statements that refer to content area during the four lessons.

Table 1: Ratio between statements related representations of MI principle and between the total statements that relate to content area.

\begin{tabular}{lllll}
\hline & Infinity & Identification & Peano's axiom & Formulation \\
\hline Ratio & 0.2025 & 0.2982 & 0.1237 & 0.3756 \\
\hline
\end{tabular}

Thus, we may summarize the main results of the teaching process which is based on the professor's perceptions of the MI principle:

The teacher professor began to emphasize the perception "MI is a jump to infinity": during the first two lessons, figure (2a) and figure (2b). this perception is about $20 \%$ of the professor's references, table 1 . The following excerpt, from the first lesson, make sense to this conception:

\section{Excerpt 1: A jump to infinity}

Does the set $\{1,2,3,4,6,7, \ldots\}$ is the set of all natural numbers?

$\ldots$

I can verify for any finite number, if it belongs to the set or not, but I can't do this for all the numbers, because this needs an infinite time; actually, a problem like this demands a jump from finite to infinite.

It is clear that the professor represent the MI principle as "a jump to infinity".

The perception "MI principle is an axiom of Peano" took place about 13\% of the total statements according to the professor's representation during the first lesson, increased to about $29 \%$ during the second lesson and decreased to $2 \%$ during the third lesson. Excerpt 2 is an example to this kind of statements

\section{Excerpt 2: MI principle is an axiom of Peano}

1. There exist a first number $1 \in N$

2. for each $x$ there is one successor number, $x^{\prime}$.

3. $x^{\prime} \rightarrow>1$, i.e. there exists no number whose successor is 1 .

4. If $x^{\prime}=y^{\prime} \rightarrow x=y$ i.e. if successor numbers are equal so the original numbers are equal too.

5. An identification axiom: let $S \rightarrow N$, such that
i) $1 \in S$ and
ii) if $x \in S$ then $x+1 \in S$ then $S=N$. 
"MI principle identifies the natural number set" had main weights in the first and third lessons, but also it approached to $20 \%$ of the professor's references during the second lesson, figure(2a), (2b) and (2c). Moreover, this perception was about $30 \%$ of the professor's whole representation the MI principle, table 1. Excerpt 3 makes sense to this professor's conception the MI principle.

\section{Excerpt 3: MI principle identifies the natural number set}

Virtually, I work with a set, and I want to know if this set is the set of all natural numbers

"The formulation of MI principle" had about $38 \%$ of the total references to the various conceptions according to the professor's lessons, table 1 . But, the professor did not refer to this conception during the first and second lesson, figure (2a) and (2b). While in the third lesson our subject referred to this conception about $36 \%$, and in the fourth lesson this conception was the main one. The following excerpt is an example to the professor's formal representation of the MI principle.

\section{Representation 4: The formulation of the MI principle}

Let $S \subset N$ with the properties i) $1 \in S$ and ii) if $x \in S$ then $x+1 \in S$ then $S=N$.

\section{The learning process - Students' data analysis}

The learning process was investigated by examining the STM and LTM of the MI principle among the teacher professor's students. As was mentioned, the students' data mainly relied on the analysis of the students' answers to the questionnaire. To answer the questionnaire's questions, the students have to apply their acquired knowledge, not simply repeat what they have read in the textbook or solve a MI problem routinely. Table 2 summarizes the results of the students' answers in both classes (11th and 12th grades) to the first and the second questions of the questionnaire.

The results in table 2, show that most of the answers to Question 1 were the correct answer Part (c), i.e. mathematical induction relates to the set of natural numbers as order; while a negligible percent of the 11th graders gave the wrong answer Part (a) (MI relates to natural numbers as the sum operation) and about $12 \%$ of 12th graders referred to the same answer. The essential meaning of this result is that the professor was able to internalize the correct relation into his students.

The answers to Question 2 show that there is a difference between the distributions of the answers of the 11 th grade students and those of the 12th grade students. $67 \%$ of 12 th graders and only about $15 \%$ of 11 th graders perceive correctly that "MI characterizes the set of natural numbers", while $58.5 \%$ of 11 th graders and only $9 \%$ of 12 th graders perceive that "MI defines the set of natural numbers", table 2; moreover, $24 \%$ of 11 th graders and $9 \%$ of 12 th graders decided that all the suggested answers were wrong. To examine the reason for this result, further inquiry was done; this inquiry showed that 10 of 24 students from the 11th grade did ask about the meaning of "define" and "characterize" because they did not understand the difference between the two expressions. Thus, this may explain the differences between the two distributions as a function of class and we may claim that the students confused between the two expressions especially 11th graders, moreover, in the LTM the students tended to understand the expressions and more students perceive correctly the function of MI principle.

Table 2: Distribution of the answers to the first two questions in the induction questionnaire.

\begin{tabular}{lcccccccc}
\hline The answer's & \multicolumn{2}{c}{ Part a } & \multicolumn{2}{c}{ Part b } & \multicolumn{2}{c}{ Part c } & \multicolumn{2}{c}{ Part d } \\
\cline { 2 - 9 } percentage & $\mathbf{1 1}^{\text {th }}$ grade & $\mathbf{1 2}^{\text {th }}$ grade & $\mathbf{1 1}^{\text {th }}$ grade & $\mathbf{1 2}^{\text {th }}$ grade & $\mathbf{1 1}^{\text {th }}$ grade & $\mathbf{1 2}^{\text {th }}$ grade & $\mathbf{1 1}^{\text {th }}$ grade & $\mathbf{1 2}^{\text {th }}$ grade \\
\hline Question 1 & $2.4 \%$ & $12 \%$ & $0 \%$ & $0 \%$ & $97.6 \%$ & $88 \%$ & $0 \%$ & $0 \%$ \\
Question 2 & $58.5 \%$ & $9.1 \%$ & $14.6 \%$ & $67 \%$ & $0 \%$ & $12 \%$ & $24.4 \%$ & $9.1 \%$ \\
\hline
\end{tabular}

Completing the questionnaire analysis, let us present the results which were obtained from the analysis of question 3, this question reveals different conceptions of MI principle among the students. The answers to Question 3 were divided into categories as the students' perceptions of the MI principle. We get seven categories including the formulation of MI principle as perceived by the student.

Let us present all the categories including examples highlighting the students' answers; for each category we present also the distribution of the students' answers: 11th grade, 12th grade and total respectively: 
Category i: Mathematical induction is a jump to infinity; $(0 \%, 3 \%, 0.9 \%)$.

Example:

"Mathematical induction is a means of transferring from the finite to the infinite."

Category ii: Mathematical induction is an axiom, including its exact formulation; $(0 \%, 3 \%, 0.9 \%)$.

Example:

"let $S$ be the set of all natural numbers $n$ that satisfies

...

we have to prove that $S=N$. the proof is by induction

1. $1 \in S$

2. suppose that $x \in S$, we have to prove that $x \in S$, e.g. proof:

...

according to the mathematical induction axiom $S=N . "$

Category iii: Mathematical induction is an axiom, including incorrect formulation; $(15 \%, 15 \%, 15 \%)$.

Example:

" $x+1 \in S$ according to the mathematical induction axiom $S=N$."

Category iv: Mathematical induction characterizing the natural numbers' set, including exact formulation; $(56 \%, 76 \%, 65 \%)$.

Example:

"Let $S$ be a subset of natural numbers $x$ that satisfies

If i) $1 \in S$, and ii) suppose that $x \in S \rightarrow x+1 \in S$ then $S=N$."

Category v: Mathematical induction characterizes the natural numbers, including the exact formulation and its extension to sets that are equivalent to the natural numbers set; ( $5 \%, 0 \%, 3 \%)$.

\section{Example:}

If we want to prove a proposition for all the numbers $n=1,2,3, \ldots$

(if the proposition is for the set $n=2,4,6, \ldots$ so we have to carry out a transformation so that the proposition will be true or the natural numbers)

let $S$ be the set of natural numbers $x$ that

1. $1 \in S$, substitute 1 and prove the preposition

2. suppose that $x \in S$ and prove that $x+1 \in S$ so according to the mathematical induction axiom $S=N$."

Category vi: Mathematical induction characterizes the natural numbers set, including an inexact formulation; ( $22 \%, 3 \%, 14 \%)$.

\section{Example:}

"prove that for all natural number $n$ exists:

...

let $S$ be the set of natural numbers $x$ that satisfies

$S \rightarrow N$ we'll prove that $S=N$

Suppose that

....

Prove:

$x \in S$

$x=N$

$N=S^{\prime \prime}$

Category vii: Do not know/ cannot remember; $(2 \%, 0 \%, 0.9 \%)$. 


\section{Example:}

\section{"I don't remember."}

The results show that most of the students in our study perceive the mathematical induction principle as: "mathematical induction identifies the natural numbers set". Some added a rigorous formulation of the axiom and others added an inexact formulation.

\section{Teaching - Learning data: Triangulation}

Methodological triangulation of the previous teaching process and learning process reveals to the following results.

1. "MI relates to the set of natural numbers as order" perception: During the first two lessons the professor referred to the axioms of Peano (about 13\% and 29\% of the first and second lessons respectively). The analysis of the learning process showed that in the STM and LTM, most of the professor's students (about $98 \%$ and $88 \%$ in the STM and LTM respectively) perceived that "MI relates to the set of natural numbers as order". Thus, we may claim that the students were influenced powerfully by the teacher professor's conception which was expressed via the axioms of Peano.

2. "MI is a jump to infinity" perception: During the first two lessons the teacher professor emphasized the property "MI is a jump to infinity", the previous teaching process analysis revealed that the professor referred to this conception about $20 \%$ of all his references. While the learning process analysis indicated that a negligible percent of the professor's students had this perception.

3. "MI is an axiom of Peano's axioms" perception: In general the professor referred to this conception about $10 \%$ of his total references to the MI principle. But, he referred to this conceptions powerfully only during the second lesson, and also he was used to write the expression "according to the mathematical induction principle..." during practicing the principle. The learning process showed that the students perceive "MI is an axiom of Peano" as is, was rarely; but about 3\% and 15\% (STM and LTM respectively) used the expression "according to the mathematical induction principle" including the correct formulation and the incorrect formulation. Thus, we may conclude that toward LTM, a moderate percentage of the students developed this perception of the MI principle. Moreover we may claim that this perception was created as a result of solving (accurately or non-accurately) a large number of MI problems in the form of the professor's formulation.

4. "MI characterizing the natural numbers' set" perception: This perception had the biggest weight during the professor's lessons, beginning with 0.31 as a weight in the first lesson and ending with about 0.58 in the third lesson. The professor emphasized the power of MI principle by repeating the statement "I work with a set, and I want to know if this set is the set of all natural numbers". This perception was examined among the professor's students by the second and the third questions of the questionnaire. On the one hand, the main result according to the second question showed that toward LTM the students tended to perceive MI as characterizing the set of natural numbers; but in the STM only 15\% of students had this perception of the MI, table 2. On the other hand, the third question analysis yielded that the professor's students perceived the MI principle as characterizing the set of natural numbers at a great extent $(65 \%$ of all the students in both groups) especially in the LTM (76\% of the 12th graders).

5. "Formulation of the MI principle" perception: The professor emphasized the MI formulation during the third and fourth lessons; during the fourth lesson the professor concentrated only on this perception. The professor's students perceived correctly the MI principle in its formulation form powerfully, $56 \%$ at STM and $76 \%$ at LTM, but there was a negligible percent of students who perceived the principle unaccurate.

\section{Discussion}

The influence of learning process by teaching process is not a new claim. What is innovative in this study is characterizing the students' perceptions of the MI principle and determining the powerful perception. Moreover, examining the relation between the professor's several perceptions of the MI principle and his students' conception the principle is a new idea.

Our findings show that the students' perception of MI principle essentially was: "mathematical induction identifies natural number set" (categories iv, v and vi); but not as "a method of proof which is applied to 
statements which most often figuratively come out of the sky" (Lowenthal \& Eisenberg, 1992; p.235). However, it was clear that the students referred to the formulation of the MI principle - some wrote an exact formulation and others wrote an inexact one - not as a method of proof, but accompanied with an explanation if necessary (e.g. category v). This confirms Henkin's (1961) suggestion that set-theoretical formulation has pedagogical advantages.

Further, our findings show that our subject (the professor) represented the MI principle in four different ways. These representations have a main function in building, and developing the learners' understanding (Hiebert \& Carpenter, 1992); as it was urged " for teachers to facilitate learning with understanding, they must know how to interpret or represent the mathematical ideas they wish their students to learn" (Fennema \& Franke, 1992; p. 154). Actually, a learning process had happened, the students were exposed to different representations of the MI principle. Most learners in this study stored the MI principle as "mathematical induction identifies natural number set" (including formulation) in the STM; and the findings also show that this information of knowledge was transmitted to the LTM. So this means that most of the students were influenced by the representation of the MI principle as identifying the natural number set, moreover most of them included the exact formulation of MI. Not forgetting that a small percentage of our learners had perceived the MI principle as a jump to infinity, it was only in the LTM, this means that here, too, there was a process of learning, such that the students were influenced by the examples and representations that were given by the professor. The representations of the MI principle as an axiom wasn't emphasized by the professor (table 1), and constituted only $12 \%$ of his total explanation; but some students were perceived it as an axiom including formulation. Further inquiry showed that this happened because these students were exposed to the term from another person in addition to the professor's representations.

It can be urged that the students' perceptions of a mathematical term were influenced by the several representations of this term. Moreover, it was concluded that the set-theoretical representation of MI has advantages as Henkin (1961) urged.

\section{References}

Dubinsky, E. (1986). Teaching Mathematical Induction I, The Journal of Mathematical Behavior, Vol. 5, pp. 305-317.

Dubinsky, E. (1990). Teaching Mathematical Induction II, The Journal of Mathematical Behavior, Vol. 8, No. 3, pp. 285-304.

Ernest, P. (1984). Mathematical Induction: A Pedagogical Discussion, Educational Studies in Mathematics, Vol. 15, pp. 173-179.

Fennema, E. and Franke, M.L. (1992). Teachers' Knowledge and its Impact, In Grouws D. (Ed.): Handbook of Research on Mathematics Teaching and Learning, A Project of the National Council of Teachers of Mathematics, Macmillan Publishing Company, New York, pp. 147-164.

Fischbein, E. \& Engel, I. (1989). Psychological Difficulties in understanding the Principle of Mathematical Induction, In Vergenaud, G. et al., (Eds.), Proceedings of the 13th International Conference for the Psychology of Mathematics Education, Paris, France, pp. 276282.

Hall, J. E. (1971). Algebra: A Precalculus Course, Brooks/Cole Publishing Company, Belmont, California.

Harel, G. \& Sowder, L. (1998). Students' proof schemes: Results from exploratory studies. In A. Schoenfeld, J Kaput \& E. Dubinsky (Eds.), Research in collegiate mathematics education, III (pp. 234-283).Providence: American Mathematical Society.

Harel, G. \& Sowder, L. (2007). Toward comprehensive perspectives on the learning and teaching of proof. In F. K. Lester (Ed.), The second handbook of research on mathematics teaching and learning (pp. 805-842). Washington: NCTM.

Harel, G. (2002). The Development of Mathematical Induction as a Proof Scheme: A Model for DNR Based Instruction. In S. Campbell \& R. Zazkis (Eds.) Learning and Teaching Number Theory. New Jersey: Ablex Publishing Corporation.

Henkin, L. (1961). Mathematical Induction, MAA film manual no. 1, The Mathematical Association of America. Ann-Arbor MI: Cushing-Malloy, Inc.

Hiebert, J. \& Carpenter, T. P. (1992). Learning and Teaching with Understanding, In D. A. Grouws (Ed.): Handbook for Research on Mathematics Teaching and Learning (pp. 65-97). NY: Macmillan Publishing Company.

Lowenthal, F. and Eisenberg, T. (1992). Mathematical Induction in School: An Illusion of Rigor, School Science and Mathematics, Vol. 92(5), pp. 233-238.

Maxfield, J. E. \& Maxfield, M. W. (1971). Abstract Algebra - and Solution by Radicals, W.B. Saunders Company Philadelphia, London, Toronto.

Movshovitz-Hadar, N. (1993). Mathematical Induction: A Focus on the Conceptual Framework, SchoolScience and Mathematics, Vol. 93(8), pp. 408-417.

National Council of Teachers of Mathematics (NCTM) (2000). Principals and standards for school mathematics. Reston, VA: National Council of Teachers of Mathematics.

Palla, M., Potari, D., \& Spyrou, P. (2012). Secondary Sschool Students' Understanding of Mathematical Induction: Structural Characteristics and the Process of Proof Construction. International Journal of Science and Mathematics Education, 10(5), $1023-1045$.

Pedemonte, B. (2007). How can the relationship between argumentation and proof be analysed? Educational Studies in Mathematics, 66, 23-41.

Schoenfeld, A. H. (1985). Mathematical problem solving. Orlando (FL): Academic Press.

Sfard, A. (1998). On two metaphors for learning and on the danger of choosing just one. Educational Researcher 27(2), pp.4-13. 
Sfard, A. (2001). There is more to discourse than meets the ears: Looking at thinking as communicating to learn more about mathematical learning. Educational Studies in Mathematics, 46, pp. 16-57.

Sharif-Rasslan, A. (1999). A Professional Mathematician as a High School Teacher - A Case Study, Unpublished doctoral dissertation, Technion - Israel Institute of Technology.

Silver, E. (1987). Foundations of Cognitive Theory and Research for Mathematics Problem-Solving .In A. H. Schoenfeld (ed.): Cognitive Science and Mathematics Education. USA, Lawrence Elbaum Associates, Inc., pp. 33-60.

Yin, R. K. (2003). Case study research: Design and methods (3rd ed.). Thousand Oaks, CA: Sage. 